Article

\title{
Size-Controlled and Optical Properties of Monodispersed Silver Nanoparticles Synthesized by the Radiolytic Reduction Method
}

\section{Elias Saion $^{1, *}$, Elham Gharibshahi ${ }^{1}$ and Kazem Naghavi ${ }^{2}$}

1 Physics Department, Universiti Putra Malaysia, UPM Serdang 43400, Selangor, Malaysia; E-Mail: elhamgs2002@yahoo.com

2 Department of Science, Kerman branch, Islamic Azad University, Kerman 76351-31167, Iran; E-Mail: kazem.naghavi@gmail.com

* Author to whom correspondence should be addressed; E-Mail: elias@science.upm.edu.my; Tel.: +603-8946-6654; Fax: +603-8945-4454.

Received: 20 October 2012; in revised form: 21 March 2013 / Accepted: 22 March 2013 / Published: 11 April 2013

\begin{abstract}
Size-controlled and monodispersed silver nanoparticles were synthesized from an aqueous solution containing silver nitrate as a metal precursor, polyvinyl alcohol as a capping agent, isopropyl alcohol as hydrogen and hydroxyl radical scavengers, and deionized water as a solvent with a simple radiolytic method. The average particle size decreased with an increase in dose due to the domination of nucleation over ion association in the formation of the nanoparticles by gamma reduction. The silver nanoparticles exhibit a very sharp and strong absorption spectrum with the absorption maximum $\lambda_{\max }$ blue shifting with an increased dose, owing to a decrease in particle size. The absorption spectra of silver nanoparticles of various particle sizes were also calculated using a quantum physics treatment and an agreement was obtained with the experimental absorption data. The results suggest that the absorption spectrum of silver nanoparticles possibly derived from the intra-band excitations of conduction electrons from the lowest energy state $(n=5$, $l=0)$ to higher energy states $(n \geq 6 ; \Delta l=0, \pm 1 ; \Delta s=0, \pm 1)$, allowed by the quantum numbers principle. This demonstrates that the absorption phenomenon of metal nanoparticles based on a quantum physics description could be exploited to be added into the fundamentals of metal nanoparticles and the related fields of nanoscience and nanotechnology.
\end{abstract}

Keywords: theory of metal nanoparticles; shell model; particle size; absorption maxima; conduction band; silver nanoparticles; radiolytic synthesis 


\section{Introduction}

Ancient civilizations used metal nanoparticles for their brilliant colors, and they can be found in the stained glass windows of the Middle Ages, and yet, they continue to attract considerable attention today [1-3]. They exhibit size-dependent properties such as the tuning of absorption energy with particle size, a blue shift of absorption onset, and an enhancement of photocatalytic activities with a decrease in particle size. These novel properties are the consequence of a large number of surface atoms and three-dimensional quantum confinement of electrons, which are believed to be the factors regulating the physical and chemical properties of metal nanoparticles. However, a number of fundamental issues of metal nanoparticles have to be addressed. For instance, metal nanoparticles display a sharp absorption spectrum when illuminated with UV-visible light and, in this situation; they can perform as photocatalysts to boost a chemical reaction. Hence, it seems that the catalysts' activity is directly linked to the action of conduction electrons, which provide the absorption spectrum of the metal nanoparticles. Because of their size in the nanoscale regime between the atomic and bulk structures, metal nanoparticles may serve as a model system that possesses both the quantum and classical physics principles, which may be used to describe their behaviors. Which of these principles could interpret accurately the behaviors of metal nanoparticles?

Among metallic nanoparticles, a noble silver (Ag) nanoparticle continues to be interesting nanomaterial in nanoscience owing to their excellent optical and electronic properties [4-6]. The research on this material has steered into many fields: catalysis [7], electrochemical sensors [8,9], antibacterial activity [10,11], degradation of environmental pollutants [12], biological labeling [13], drug delivery [14], surface-enhanced Raman scattering [15], and cancer therapy [16]. The optical properties of Ag nanoparticles are dictated by the geometrical parameters such as size and shape [17]. In recent years, much effort has been focused on the development of new strategies for the synthesis of $\mathrm{Ag}$ nanoparticles of high dispersion and uniform size and shape. They fall under two categories, i.e., the chemical and physical approaches. The chemical approach utilizes several techniques such as the chemical, electrochemical, sol-gel, microemulsions, reverse micelles, and polyol methods [18-20]. To produce small clusters or aggregates of Ag nanoparticles, silver ions in aqueous solution were commonly reduced using a reducing agent, such as sodium borohydride [21], hydrazine [22], tetrabutylammonium borohydride [23], sodium citrate [24], dimethylformamide [25], ascorbic acid [26], and alcohols or polyols [27,28]. In the physical approach, the Ag ions in aqueous solution may be reduced by means of several irradiation techniques, including ultraviolet light [29,30], microwave [31], ultrasound [32], laser ablation [33], and gamma irradiation [34], without the need for a reducing agent.

Among the physical techniques, the synthesis of Ag nanoparticles using the radiolytic method has not been completely explored. Previous works emphasized only the mechanism of reduction and aggregation of $\mathrm{Ag}$ nanoparticles [35-38]. Nevertheless, this technique continues to receive considerable interest [39-42]. The method offers several advantages over the conventional methods: the process is simple and clean; controlled reduction of metal ions can be carried out without using a reducing agent or producing undesired oxidation products; it provides metal nanoparticles in a fully reduced, highly pure and highly stable state; it is harmless and environmentally friendly; it has proven to be a powerful method of synthesis at room temperature; and, it produces monodispersed 
nanoparticles [35-43]. The present study aims at examining the effect of dose on particle size of Ag nanoparticles and to interpret the optical properties with a new theory of metal nanoparticles based on quantum physics, which could add to the fundamental knowledge of metal nanoparticles and the related fields of nanoscience and nanotechnology.

\section{Results and Discussion}

\subsection{Formation of Ag Nanoparticles in Colloidal PVA}

The interaction of gamma photons with matter involves several distinct processes depending on the energy of the photons and on the density and atomic number of the medium. $1.25-\mathrm{MeV}{ }^{60} \mathrm{Co}$ gamma rays interact with matter in aqueous solution by photoelectric absorption, Compton scattering, and pair production, resulting in the formation of secondary electrons, which is mostly coming from the Compton scattering effect. These free and energetic electrons can induce several reactive species such as hydrated electrons $\left(\mathrm{e}^{-}\right.$aq $)$, hydroxyl radicals $\left(\mathrm{OH}^{\circ}\right)$, and hydrogen radicals $\left(\mathrm{H}^{\circ}\right)$ by radiolysis of water (Reaction 1).

$$
\mathrm{H}_{2} \mathrm{O} \stackrel{\gamma \text {-rays }}{\longrightarrow} \mathrm{e}_{\mathrm{aq}}^{-}, \mathrm{H}_{3} \mathrm{O}, \mathrm{H}^{\bullet}, \mathrm{OH}^{\bullet}, \mathrm{H}_{2}, \mathrm{H}_{2} \mathrm{O}_{2}, \mathrm{H}_{3} \mathrm{O}^{+} \quad \text { (radiolysis of water) }
$$

The formation of Ag nanoparticles by gamma radiolytic method can be divided into two steps. First occurs the formation of the atom by the nucleation process; this is followed by the formation of nanoparticles by the aggregation process. The nucleation process can be described by the following reactions. The hydrated electrons $\left(\mathrm{e}^{-}\right.$aq $)$are a strong reducing agent and can reduce $\mathrm{Ag}$ ions $\left(\mathrm{Ag}^{+}\right)$into zero-valent $\mathrm{Ag}$ atoms $\left(\mathrm{Ag}^{0}\right)$ (reaction 2) [35]. In our case, the $\mathrm{Ag}$ ions, $\mathrm{Ag}^{+}$come from silver nitrate in an aqueous solution of PVA used as a stabilizing agent to restrict the agglomeration of reduced $\mathrm{Ag}$ atoms, thus limiting the aggregation and the size of $\mathrm{Ag}$ nanoparticles.

$$
\mathrm{Ag}^{+}+\mathrm{e}_{\mathrm{aq}}^{-} \rightarrow \mathrm{Ag}^{0} \quad \text { (nucleation) }
$$

The $\mathrm{H}^{\bullet}$ and $\mathrm{OH}^{\bullet}$ radicals formed in radiolysis of water, (Reaction 1) are also strong reducing agents in aqueous solution. To avoid this oxidation, the radicals were scavenged by adding isopropanol (IPA). The $\mathrm{H}^{\circ}$ and $\mathrm{OH}^{\bullet}$ radicals can react with IPA molecules during and after irradiation to form isopropanol radicals (IPA) (Reactions 3 and 4), which then react with $\mathrm{Ag}$ ions, $\mathrm{Ag}^{+}$to form zero-valent metal atoms, $\mathrm{Ag}^{0}$ (Reaction 5). However, the reduction of $\mathrm{Ag}$ ions, $\mathrm{Ag}^{+}$by hydrated electrons is the main process for the formation of $\mathrm{Ag}$ nanoparticles under gamma irradiation [44].

$$
\begin{gathered}
\mathrm{H}^{\bullet}+\mathrm{CH}_{3}-\mathrm{CH}(\mathrm{OH})-\mathrm{CH}_{3} \rightarrow \mathrm{CH}_{3}-\mathrm{C}^{\bullet}(\mathrm{OH})-\mathrm{CH}_{3}+\mathrm{H}_{2} \quad \text { (radicals formation) } \\
\mathrm{OH}^{\bullet}+\mathrm{CH}_{3}-\mathrm{CH}(\mathrm{OH})-\mathrm{CH}_{3} \rightarrow \mathrm{CH}_{3}-\mathrm{C}^{\bullet}(\mathrm{OH})-\mathrm{CH}_{3}+\mathrm{H}_{2} \mathrm{O} \quad \text { (radicals formation) } \\
\mathrm{Ag}^{+}+\mathrm{CH}_{3}-\mathrm{C}^{\bullet}(\mathrm{OH})-\mathrm{CH}_{3} \rightarrow \mathrm{CH}_{3}-\mathrm{CO}-\mathrm{CH}_{3}+\mathrm{H}^{+}+\mathrm{Ag}^{0} \quad \text { (nucleation) }
\end{gathered}
$$

The formation of Ag nanoparticles by the aggregation process can be described by the following reactions. The zero-valent $\mathrm{Ag}^{0}$ finally coalesce to form aggregates $\mathrm{Ag}^{0}{ }_{\mathrm{m}+1}$ (Reaction 6). The agglomerated $\mathrm{Ag}$ nanoparticles, $\mathrm{Ag}^{0}{ }_{\mathrm{m}+1}$ can also combine with $\mathrm{Ag}^{+}$to form larger $\mathrm{Ag}$ ions, $\mathrm{Ag}^{+}{ }_{\mathrm{m}+2}$ (Reaction 7), which can then be reduced by hydrated electrons into larger $\mathrm{Ag}$ nanoparticles, $\left(\mathrm{Ag}_{\mathrm{m}+2}{ }^{2}\right)$ (Reaction 8). The binding energy between two Ag atoms is stronger than the Ag-PVA bond 
energy [45]. Hence, when the Ag atoms stumble upon each other, they are attracted and diffused into a larger $\mathrm{Ag}$ nanocluster, $\mathrm{Ag}^{0}{ }_{\mathrm{m}+\mathrm{n}}$ (Reaction 9).

$$
\begin{array}{cc}
\mathrm{Ag}^{0}{ }_{\mathrm{m}}+\mathrm{Ag}^{0} \rightarrow \mathrm{Ag}^{0}{ }_{\mathrm{m}+1} & \text { (agglomeration) } \\
\mathrm{Ag}^{0}{ }_{\mathrm{m}+1}+\mathrm{Ag}^{+} \rightarrow \mathrm{Ag}^{+}{ }_{\mathrm{m}+2} & \text { (ion association) } \\
\mathrm{Ag}^{+}{ }_{\mathrm{m}+2}+\mathrm{e}_{\mathrm{aq}}^{-} \rightarrow \mathrm{Ag}^{0}{ }_{\mathrm{m}+2} & \text { (agglomeration) } \\
\mathrm{Ag}^{0}{ }_{\mathrm{m}}+\mathrm{Ag}_{\mathrm{n}} \rightarrow \mathrm{Ag}^{0}{ }_{\mathrm{m}+\mathrm{n}} & \text { (agglomeration) }
\end{array}
$$

To prevent an increase in cluster size, a polymer is commonly used. The polymer is adsorbed on the clusters' surface to reduce the surface tension [46]. In this experiment, PVA was adopted. In aqueous medium, PVA radicals (PVA') might also be formed by PVA reacting with $\mathrm{OH}^{\bullet}$ radicals that were not scavenged by IPA. However, the $\mathrm{PVA}^{\circ}$ then react with each other to initiate an intra- or inter-molecular crosslinking between PVA chains, resulting in a three-dimensional PVA network (Reaction 10) [47], which encircles the Ag nanoparticles [48]. The adsorption of PVA network on the surface of Ag nanoparticles can reduce the surface energy and decrease further the agglomeration process of Ag nanoparticles [49]. This increases the degree of polymer capping on the surface of nanoparticles leading to smaller particle sizes.

$$
\mathrm{PVA}^{\bullet}+\mathrm{PVA}^{\bullet} \rightarrow \mathrm{PVA}^{\mathrm{PVA}} \quad \text { (crosslinked polymer) }
$$

The average particle size of $\mathrm{Ag}$ nanoparticles was determined from the photon cross correlation spectroscopy (PCCS) measurements using the cumulative distribution at 90\% [50] as shown in Figure 1. The PCCS distribution depends on the average size of the nanoparticles. It gives a short tailing effect at low percentage of cumulative distribution for a narrow particle size distribution and a long tailing effect for a wide particle size distribution. Figures 2a, $3 \mathrm{a}$ and $4 \mathrm{a}$ show the transmission electron microscopy (TEM) images of Ag nanoparticles of $4.2 \times 10^{-4} \mathrm{M} \mathrm{Ag}$ ions concentration and irradiated at doses of 20, 40 and $50 \mathrm{kGy}$, respectively. One of the TEM images, Figure 2a, was purposely measured at high magnification to demonstrate the monodispersed Ag nanoparticles obtained using the radiolytic reduction method. The TEM images and TEM size distribution show a well-monodispersed spherical shape in the size range of 22-52 nm, 14-32 and 10-36 nm for 20, 40 and $50 \mathrm{kGy}$ doses, respectively. The TEM size distributions in Figures $2 b, 3 b$ and $4 b$ were obtained and the average particle sizes were determined from the histograms to be 36.0, 25.0 and $22.5 \mathrm{~nm}$ for 20, 40 and $50 \mathrm{kGy}$, respectively. The discussion on the size width that decreased with increasing dose has been debated recently [51]. The average particle sizes synthesized at doses of 20, 40, and $50 \mathrm{kGy}$ were comparable with the PCCS measurements as shown in Table 1 This suggests that a simple PCCS technique is applicable to determine particle size of colloidal Ag nanoparticles in the present size range.

\subsection{Effect of Dose}

Figure 5 shows the particles size versus the dose for Ag nanoparticles synthesized by radiolytic method. There is a competition between the nucleation and growth processes to make up $\mathrm{Ag}$ nanoparticles. The general trend is that the average particle size decreased with an increasing dose. At high doses, where the number of nucleation events is more than the number of unreduced ions, the radiolytic reduction synthesis produces smaller particle sizes. At low doses, however, where the 
nucleation concentration is less than the concentration of unreduced ions, the zero-valent agglomerated atoms can be ionized by unreduced ions and later reduced by hydrate electrons to produce even larger Ag nanoparticles (Reactions 6 and 7) [39]. The figures illustrate that the largest particle size is seen at the lowest dose of $10 \mathrm{kGy}$, and the smallest particle size is seen at the highest dose of $70 \mathrm{kGy}$. The average particle sizes were found to be 43.9 and $15.0 \mathrm{~nm}$ for 10 and $70 \mathrm{kGy}$, respectively. We expect that by increasing the precursor concentration, the particle size would become larger due to higher metal ions concentration, thus allowing more nucleation and aggregation processes to take place in the formation of Ag nanoparticles.

Figure 1. Cumulative distribution of particle size of monodispersed Ag nanoparticles synthesized from $\mathrm{AgNO}_{3}$ concentration of $4.2 \times 10^{-4} \mathrm{M}$ in $5 \mathrm{wt} \%$ PVA at different absorbed doses of (1) 10, (2) 20, (3) 30, (4) 40, (5) 50, (6) 60, and (7) $70 \mathrm{kGy}$.

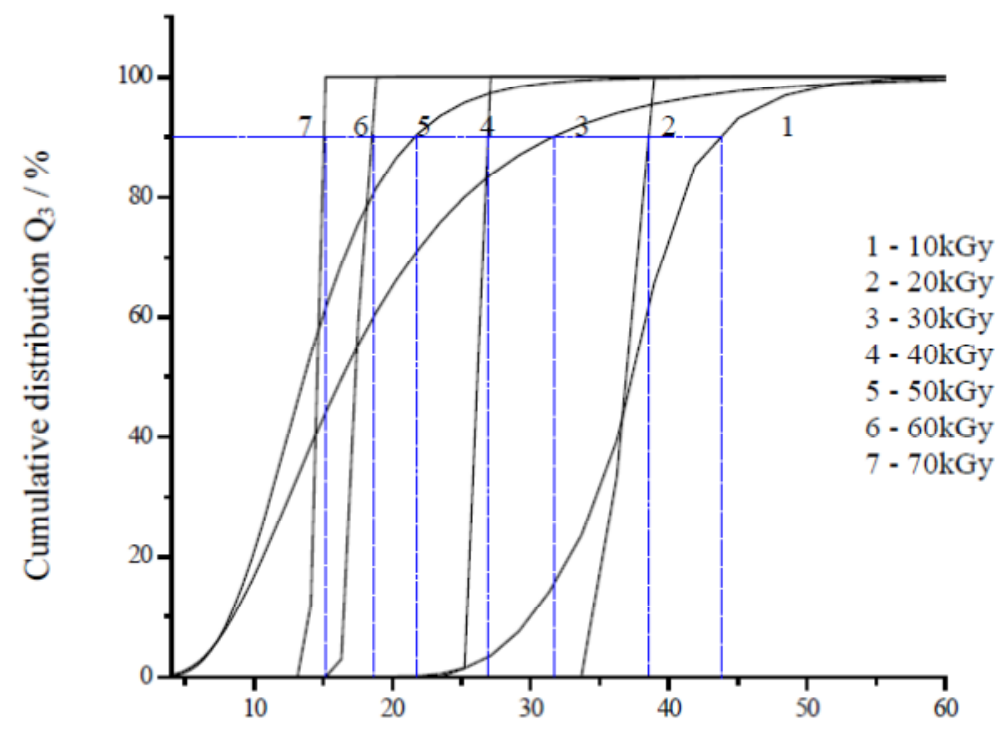

Particle size / nm

Figure 2. (a) TEM images and (b) TEM size distribution and Gaussian fitting of monodispersed $\mathrm{Ag}$ nanoparticles synthesized from $\mathrm{AgNO}_{3}$ concentration of $4.2 \times 10^{-4} \mathrm{M}$ in $5 \mathrm{wt} \%$ PVA and irradiated at $20 \mathrm{kGy}$.

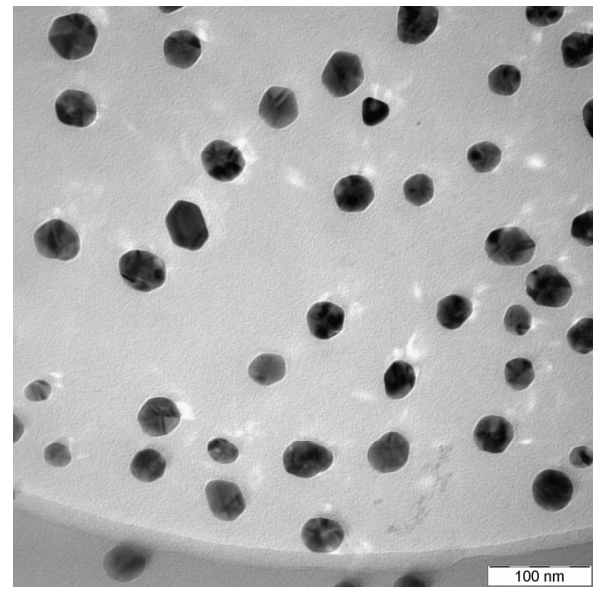

(a)

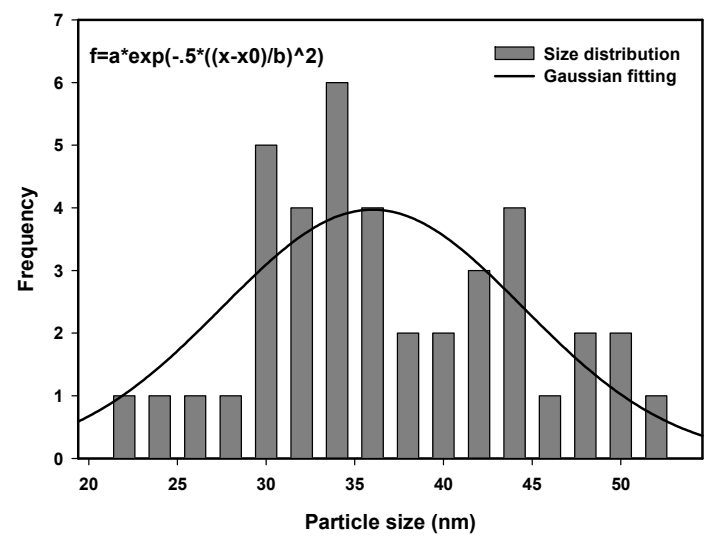

(b) 
Figure 3. (a) TEM images and (b) TEM size distribution and Gaussian fitting of monodispersed $\mathrm{Ag}$ nanoparticles synthesized from $\mathrm{AgNO}_{3}$ concentration of $4.2 \times 10^{-4} \mathrm{M}$ in $5 \mathrm{wt} \%$ PVA and irradiated at $40 \mathrm{kGy}$.

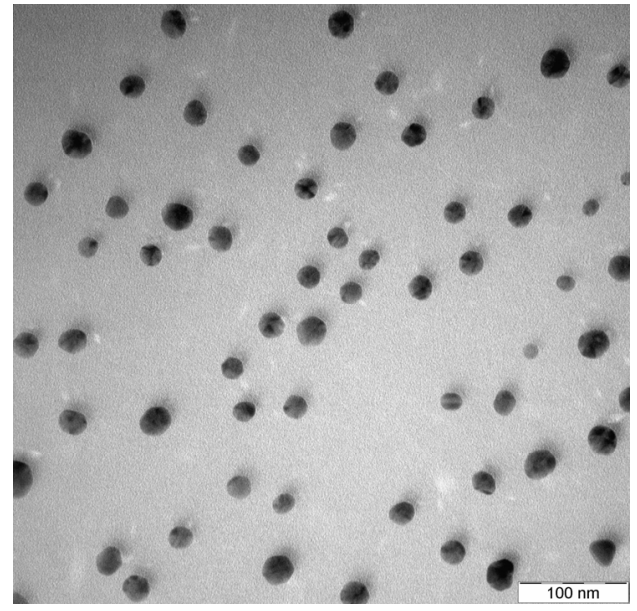

(a)

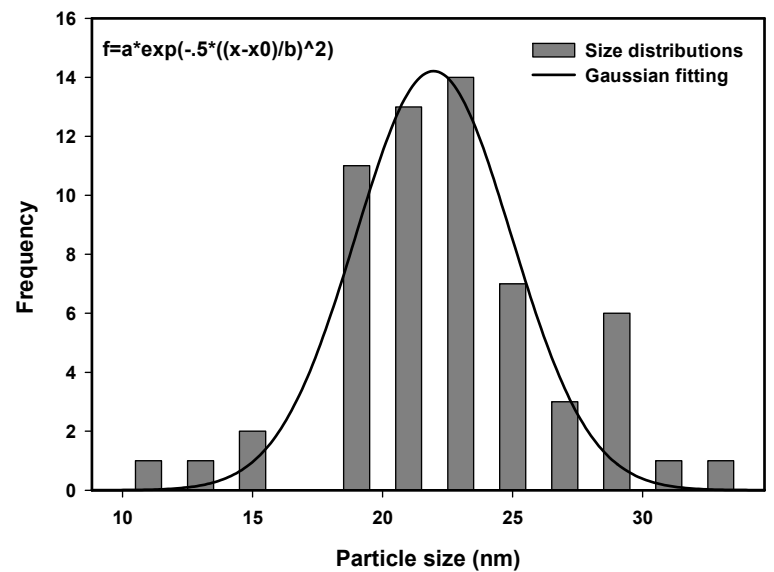

(b)

Figure 4. (a) TEM images and (b) TEM size distribution and Gaussian fitting of monodispersed $\mathrm{Ag}$ nanoparticles synthesized from $\mathrm{AgNO}_{3}$ concentration of $4.2 \times 10^{-4} \mathrm{M}$ in $5 \mathrm{wt} \%$ PVA and irradiated at $50 \mathrm{kGy}$.

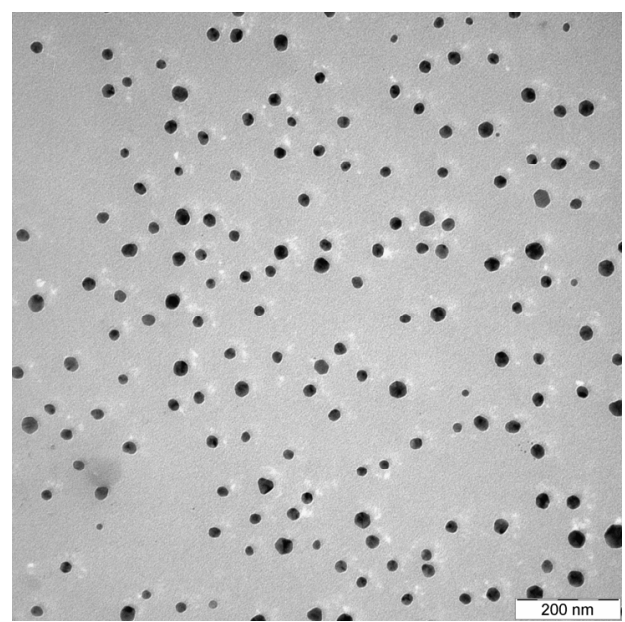

(a)

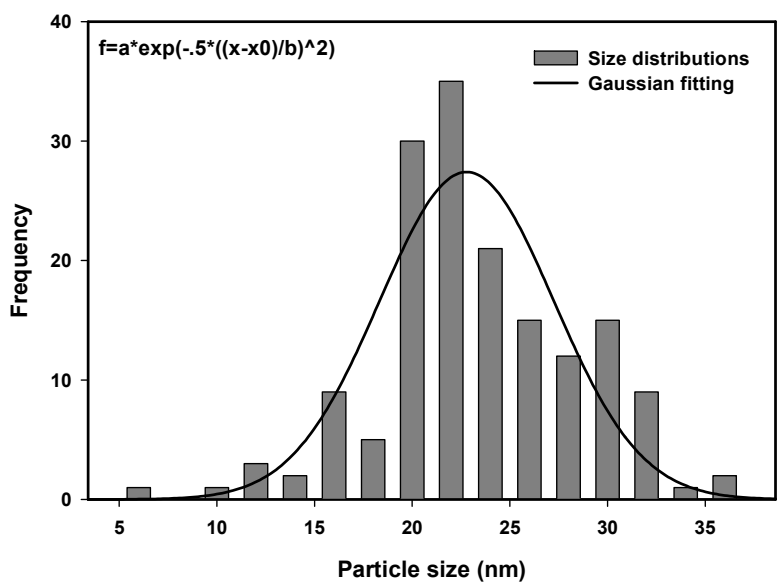

(b)

Table 1. Comparison of average particle sizes of Ag nanoparticles measured by TEM and PCCS methods for the particles synthesized from $4.2 \times 10^{-4} \mathrm{M} \mathrm{AgNO}_{3}$ in $5 \mathrm{wt} \% \mathrm{PVA}$ and irradiated with 20, 40, and $50 \mathrm{kGy}$.

\begin{tabular}{ccc}
\hline $\begin{array}{c}\text { Dose } \\
(\mathbf{k G y})\end{array}$ & $\begin{array}{c}\text { Average size measurement using } \\
\text { TEM (nm) }\end{array}$ & $\begin{array}{c}\text { Average size measurement using } \\
\text { PCCS (nm) }\end{array}$ \\
\hline 20 & 36.0 & 37.8 \\
40 & 25.0 & 26.9 \\
50 & 22.5 & 21.5 \\
\hline
\end{tabular}


Figure 5. Average particle size versus dose for $\mathrm{Ag}$ nanoparticles synthesized from $\mathrm{AgNO}_{3}$ concentrations of $4.2 \times 10^{-4}$ and $5 \mathrm{wt} \%$ PVA, showing exponential relationship between particle size and dose with dose sensitivity parameters $D_{0}$ of $74.59 \mathrm{kGy}$.

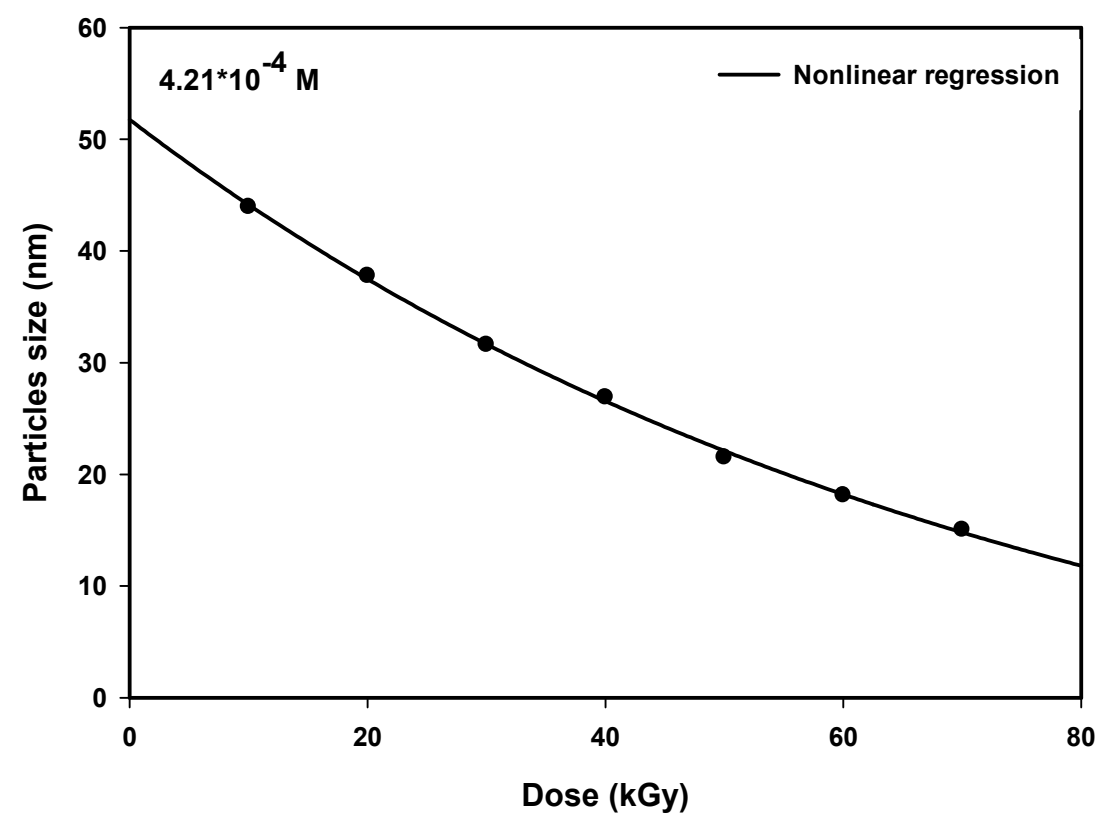

The particle size decreasing exponentially with the increasing dose may be fitted to an empirical relation of the form:

$$
d=d_{\max }+B \exp \left(-D / D_{0}\right)
$$

where $d$ is the average diameter of the nanoparticle at dose $D ; d_{\max }$ is the nanoparticle diameter at the highest dose of $70 \mathrm{kGy} ; d_{0}$ is the nanoparticle diameter at the lowest dose of $10 \mathrm{kGy}$; $B$ is the difference between $d_{\max }$ and $d_{0}$; and $D_{0}$ is the dose sensitivity parameter for particle size formation at a given precursor concentration. The values of $D_{0}$ can be determined from the inverse of the gradient of $\ln \left[\left(d-d_{\max }\right) / \mathrm{B}\right]$ versus $D$. The dose sensitivity parameters $D_{0}$ were found to be $74.63 \mathrm{kGy}$.

\subsection{Optical Properties}

The evolution of absorption spectrum of $\mathrm{Ag}$ nanoparticles synthesized from $4.2 \times 10^{-4} \mathrm{M}$ precursor concentration and $5 \mathrm{wt} \%$ PVA with increasing dose from 10 to $70 \mathrm{kGy}$ is shown in Figure 6 . The absorbance increased with increasing dose owing to the number of Ag nanoparticles multiplied with increasing dose. The color of the colloidal solution changes from yellow to dark yellow on increasing dose due to increase in the numbers of $\mathrm{Ag}^{+}$ions that have been reduced to zero-valent $\mathrm{Ag}^{0}$ atoms. This means that the number of Ag nanoparticles of smaller sizes increased with an increasing dose. The absorption peak or absorption maximum $\lambda_{\max }$ blue shifted toward lower wavelength with increasing dose, indicating the particle size decreases as the dose increases. This is quite easy to explain when the conduction electrons that produced the absorption spectrum could be treated using a quantum physics concept. 
Figure 6. Sharp absorption spectra of Ag nanoparticles synthesized from $4.2 \times 10^{-4} \mathrm{M}$ $\mathrm{AgNO}_{3}$ concentration and $5 \mathrm{wt} \% \mathrm{PVA}$ at different doses of 10 to $70 \mathrm{kGy}$. The absorption maximum $\lambda_{\max }$ blue shifted toward lower wavelength with increasing dose owing to decreasing particle size.

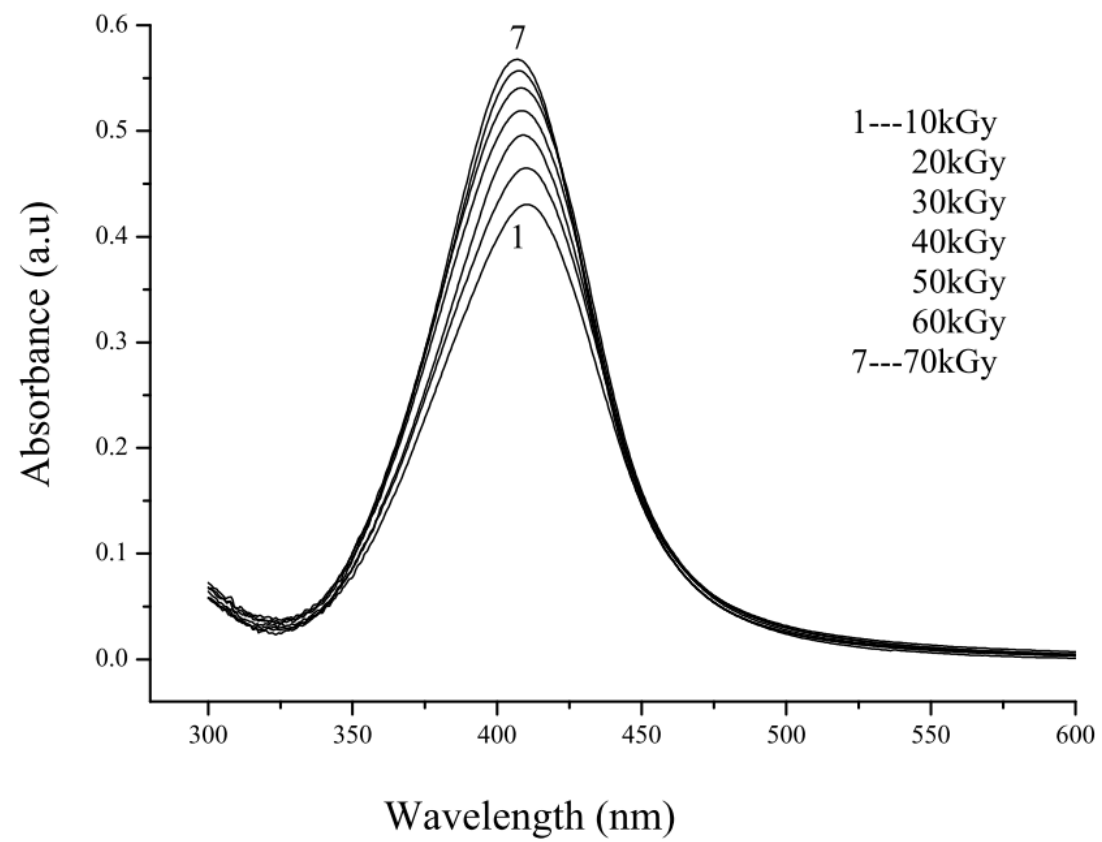

The absorption spectrum of metal nanoparticles is a well-documented phenomenon of classical physics since 1908, when the theory of particle scattering and absorbing light was first introduced by Gustav Mie [35]. It has successfully described analytically the absorption spectrum of metal nanoparticles in terms of the localized surface Plasmon resonance (LSPR), i.e., the coherent oscillation of conduction electrons on the surface of metal nanoparticles in resonance with the electromagnetic waves at the metal-dielectric interface [52-55]. For a technical understanding, however, perhaps it should be left to the experts to scrutinize the complex physics and mathematics for obtaining and interpreting the theoretical data. In quantum physics, the absorption spectrum of metal nanoparticles may be described as a result of the intra-band excitations of conduction electrons from the lowest energy state to higher energy states within the conduction band of metal nanoparticles [56,57].

Recently, we have demonstrated that the absorption maxima $\lambda_{\max }$ of platinum $(\mathrm{Pt})$ nanoparticles calculated using a new theory of metal nanoparticles agreed very well with the measured absorption maxima $\lambda_{\max }$ of radiolytic synthesized Pt nanoparticles at about 215 and $260 \mathrm{~nm}$ [58]. The theory is based on two simple assumptions: that metal nanoparticles have both geometric and electronic structures, which are both thermodynamically stable. The geometric structure may take a solid metal sphere built up from the primitive unit cells face-centered-cubic (FCC) lattice structure, and the electronic structure may follow the Jellium shell model of their respective atom. For an example, a $\mathrm{Pt}$ atom with the electronic configuration of $\mathrm{Pt}(78)$ : (Xe) $\left(5 \mathrm{~d}^{9}\right)\left(6 \mathrm{~s}^{1}\right)$ should produce a $\mathrm{Pt}$ nanoparticle with the electronic configuration of $\operatorname{Pt}(78)_{\mathrm{N}}:(\mathrm{Xe})_{\mathrm{N}}\left(5 \mathrm{~d}^{9}\right)_{\mathrm{N}}\left(6 \mathrm{~s}^{1}\right)_{\mathrm{N}}$, where $\mathrm{N}$ is the number of atoms in the nanoparticle. Using the quantum mechanics principle of quantum numbers, we have identified that the absorption peaks of 215 and $260 \mathrm{~nm}$ were derived from the intra-band excitations of conduction electrons from the lowest energy states of quantum numbers $(n=5, l=2)$ and $(n=6, l=0)$ to higher 
energy states of quantum numbers $(n \geq 6 ; \Delta l=0, \pm 1 ; \Delta s=0, \pm 1)$ and $(n \geq 7 ; \Delta l=0, \pm 1$; $\Delta s=0, \pm 1)$, respectively.

According to this model, the Ag nanoparticle can be considered an isolated solid metal sphere of diameter $d$ comprising of $N$ number of atoms that are confined in the FCC lattice structure. In solid state physics, the band theory of metals considers that outer electronic orbitals of participated atoms of nanoparticles can overlap into a conduction band occupied by conduction electrons. The electronic configuration of $\mathrm{Ag}$ nanoparticles can be categorized in spectroscopy notation as $\mathrm{Ag}(47)_{\mathrm{N}}$ : $(\mathrm{Kr})_{\mathrm{N}}$ $\left(4 d^{10}\right)_{N}\left(5 s^{1}\right)_{N}$, which is based on the Ag atom electronic configuration $\mathrm{Ag}(47)$ : $(\mathrm{Kr})\left(4 \mathrm{~d}^{10}\right)\left(5 \mathrm{~s}^{1}\right)$. For the $\mathrm{Ag}$ nanoparticle, the conduction electrons are represented by $\left(5 \mathrm{~s}^{1}\right)_{\mathrm{N}}$ valence electrons and they are considered not entirely free-contradicted by the plasmonic description-but weakly bound to the crystal backbone at the lowest energy state of quantum numbers $(n=5, l=0)$. When electromagnetic plane wave stricks Ag nanoparticle, the conduction electrons excite to higher energy states of quantum numbers ( $n \geq 6 ; \Delta l=0, \pm 1 ; \Delta s=0, \pm 1$ ), allowed by the quantum numbers principle.

The density energy functional of conduction electrons may be taken from the Thomas-Fermi-Dirac-Weizsacker model, which is the density functional theory fundamental, where the ground state electron density $\rho(\boldsymbol{r})$ is the basic variable for all ground state properties [59-64]. The Euler-Lagrangian equation $E[\rho(\boldsymbol{r})]$ can be represented as

$$
\frac{5}{3} C_{k} \int \rho(\boldsymbol{r})^{2 / 3} d \boldsymbol{r}+\frac{\eta}{8}\left[\frac{|\nabla \rho(\boldsymbol{r})|^{2}}{\rho^{2}(\boldsymbol{r})}-2 \frac{\nabla^{2} \rho(\boldsymbol{r})}{\rho(\boldsymbol{r})}\right]+v(r)+\int \frac{\rho\left(r^{\prime}\right)}{\left|r-r^{\prime}\right|} d r^{\prime}-\frac{4}{3} C_{e} \int \rho(\boldsymbol{r})^{1 / 3} d \boldsymbol{r}=E_{0}
$$

where, $\rho(r)$ is the density of conduction electrons of Ag nanoparticle, $E_{0}$ is the Fermi energy, $r$ is the displacement of conduction electrons from the center of the sphere, which is dependent on Bohr radius $a_{0}$, atomic number $Z$, and quantum numbers $n, l$, and, $s$. The first term is the Thomas-Fermi kinetic energy of homogeneous free electron gas system with $C_{k}$ as a constant $[59,60]$. The second term is the Weizsacker correction for modifying the Thomas-Fermi kinetic energy by inclusion of the exchange and correlation energy terms of inhomogeneous electron density with $\eta$ as a constant [64]. The third term is the potential energy of the system. The fourth term is the classical Coulomb potential energy of electron-electron interactions. The final term is the non-classical exchange-correlation energy containing all the remaining quantum effects not captured by the kinetic energy; the classical Coulomb potential with $C_{e}$ is the Thomas-Fermi-Dirac non-classical exchange-correlation energy constant [63]. Details of the quantum mechanical treatment can be found in our earlier publications [56,57]. It can be shown that the relation between the density and absorption may be written as $\rho \approx(Z / \sigma)^{3 / 2}$, where $Z$ is the atomic number. The transformation of density energy functional $E[\rho(r)]$ into absorption energy functional $E[\sigma(r)]$ can be calculated with a relatively simple mathematics involving only the integration and differentiation. The second order differential equation of the final absorption energy functional may be solved numerically [56,57]. All possible intra-band electronic transitions allowed would produce the same absorption maximum for a given diameter since the energy states of quantum numbers $n \geq 6$ near the Fermi level are very close to or overlap each other [56,57]. Other parameters required for the calculation of absorption spectrum of Ag nanoparticles are simply the particle size, atomic number $(Z=47)$, Fermi energy $\left(E_{0}=5.49 \mathrm{eV}\right)$ and lattice constant (0.408). 
Figure 7 show the calculated absorption spectrum of Ag nanoparticles of various diameters from 15.0 to $43.9 \mathrm{~nm}$ imitating the measured absorption spectrum of the same diameters obtained at doses of 10 to $70 \mathrm{kGy}$. Here, for each particle size, the calculated absorption spectrum is represented by one of the possible intra-band electronic transitions. It is clear that the theoretical absorption spectrum (Figure 7) and the experimental absorption spectrum (Figure 6) are not similar in terms of the height of the maximum and the width of the peak. The reason is that the calculated spectrum is based on a single $\mathrm{Ag}$ nanoparticle at a given diameter, while the measured spectrum was obtained from many $\mathrm{Ag}$ nanoparticles of the same average diameter. Figure 7 show the height of the maximum and the width of the peak of the calculated spectrum increase with increasing particle size and also the absorption maximum red shifted with increasing size. For the larger particle sizes, the number of atoms required to make up the nanoparticles is enormous, so the number of conduction electrons used in the computation is more than those of smaller particle sizes and this increases the height of the maximum and the width of the peak. The most important information between the theoretical and experimental results is that the simulated absorption maximum, $\lambda_{\max }$ consistent with the measured values as shown in Table 2. The results indicate that the absorption spectrum of Ag nanoparticles (Figure 6) most likely originated from the intra-band electronic excitations of conduction electrons from the lowest energy state of quantum numbers $(n=5 ; l=0)$ to higher energy states of quantum numbers $(n \geq 6 ; \Delta l=0, \pm 1$; $\Delta s=0, \pm 1)$ within the conduction band of $\mathrm{Ag}$ nanoparticles.

Figure 7. Theoretical absorption spectra of Ag nanoparticles for various diameters from 15.0 to $43.9 \mathrm{~nm}$ imitating the particle sizes obtained from the experiment at doses of 10 to $70 \mathrm{kGy}$. The absorption spectra are attributed to the intra-band electronic excitations of conduction electrons from the lowest energy state of quantum numbers $(n=5 ; l=0)$ to higher energy states of quantum numbers $(n \geq 6 ; \Delta l=0, \pm 1 ; \Delta s=0, \pm 1)$ allowed by the quantum numbers principle.

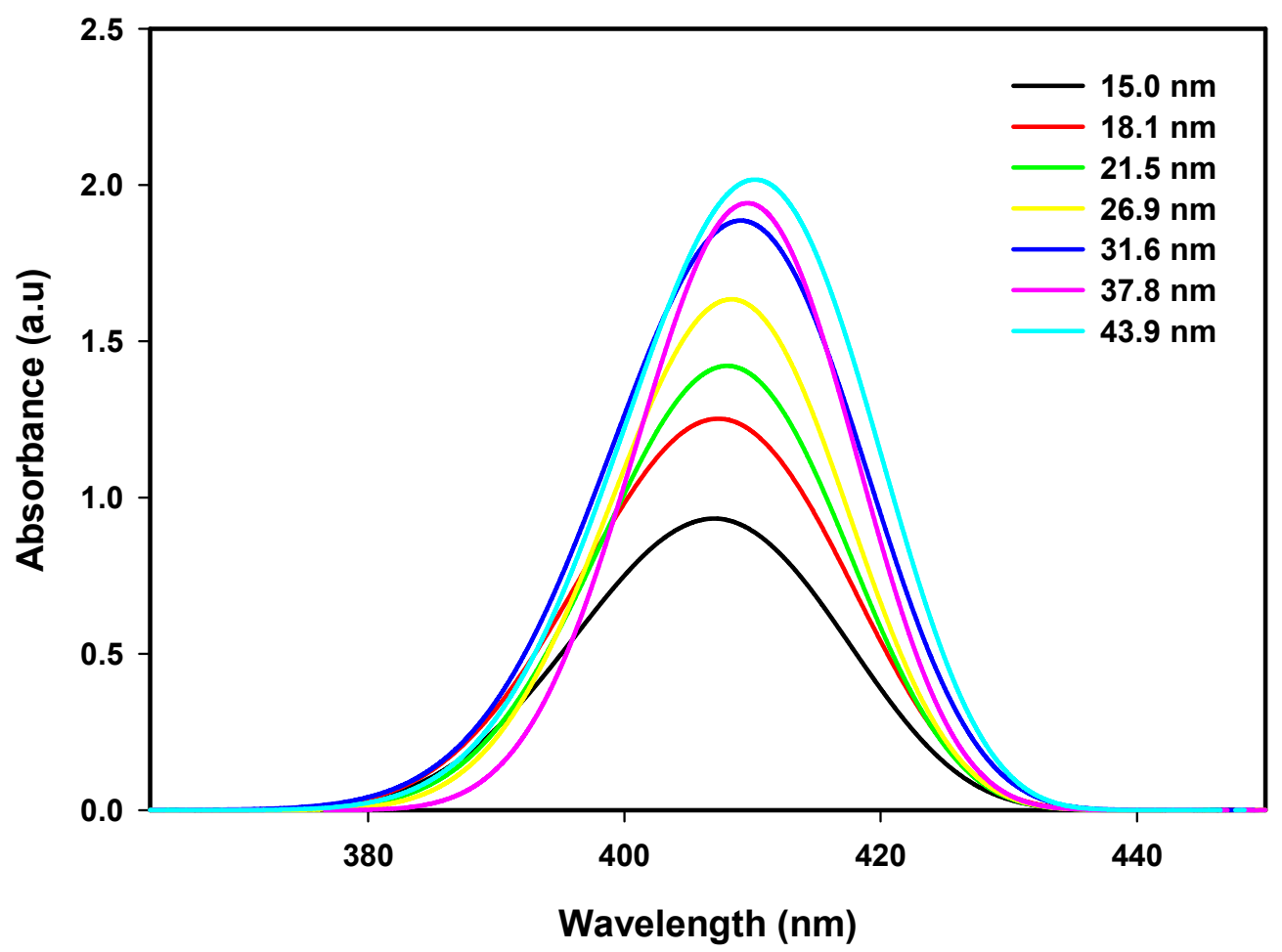


The conduction band energy, $E$ of $A g$ nanoparticles may be calculated according to $E=h c / \lambda_{\max }$, where $h$ is the Planck's constant, $c$ the speed of light, and $\lambda_{\max }$ the wavelength of the absorption maxima. The conduction band energy represents the amount of energy required to excite the conduction electrons from the lowest energy state to higher energy states influenced by the UV-visible electromagnetic radiation. Figure 8 shows the nonlinear relationship between the conduction band and the dose. The absorption maximum $\lambda_{\max }$ blue shifted toward lower wavelength from 410 to $407 \mathrm{~nm}$ with increasing dose, corresponding to conduction band of 3.023 to $3.045 \mathrm{eV}$, respectively. This is the result of decreasing particle size from $43.9 \mathrm{~nm}$ at $10 \mathrm{kGy}$ to $15.0 \mathrm{~nm}$ at $70 \mathrm{kGy}$. For smaller particles, the number of atoms to make up the nanoparticles is few, so that conduction electrons are less attracted to protons of the Ag nanoparticles and, consequently increase the conduction band and decrease the absorption peak of Ag nanoparticles.

Table 2 Average particle size, absorption maximum, and conduction band energy of $\mathrm{Ag}$ nanoparticles synthesized by radiolytic reduction method at doses of 10 to $70 \mathrm{kGy}$.

\begin{tabular}{ccccc}
\hline $\begin{array}{c}\text { Dose } \\
(\mathbf{k G y})\end{array}$ & $\begin{array}{c}\text { Particle size } \\
(\mathbf{n m})\end{array}$ & $\begin{array}{c}\text { Experiment } \\
\boldsymbol{\lambda}_{\max }(\mathbf{n m})\end{array}$ & $\begin{array}{c}\text { Theory } \boldsymbol{\lambda}_{\max } \\
(\mathbf{n m})\end{array}$ & $\begin{array}{c}\text { Conduction } \\
\text { band }(\mathbf{e V})\end{array}$ \\
\hline 10 & 43.9 & 410.0 & 410.12 & 3.023 \\
20 & 37.8 & 409.5 & 409.54 & 3.027 \\
30 & 31.6 & 409.0 & 409.12 & 3.031 \\
40 & 26.9 & 408.5 & 408.46 & 3.034 \\
50 & 21.5 & 408.0 & 408.07 & 3.038 \\
60 & 18.1 & 407.5 & 407.52 & 3.041 \\
70 & 15.0 & 407.0 & 406.80 & 3.045 \\
\hline
\end{tabular}

Figure 8. Conduction band of $\mathrm{Ag}$ nanoparticles increased nonlinearly with increasing dose owing to decreasing particle size as a result of the domination of nucleation process over ion association in the formation of nanoparticles at higher doses.

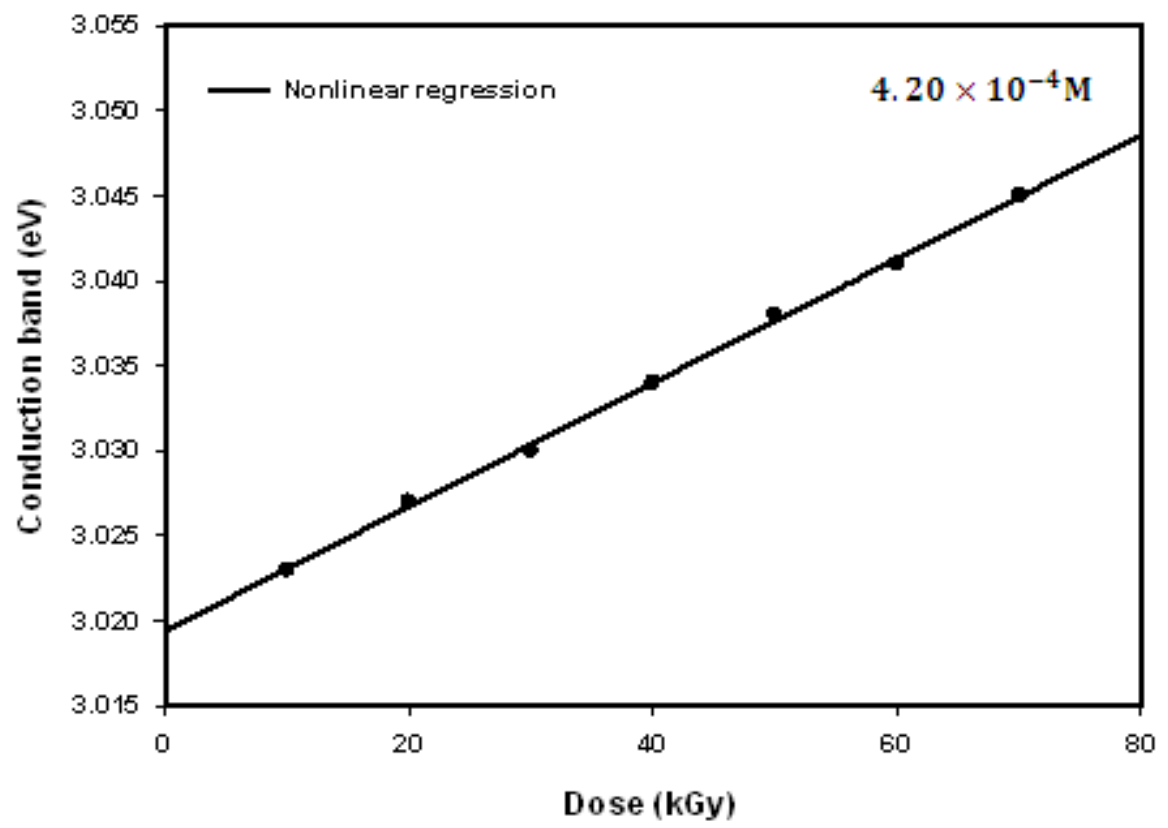


Figure 9 shows that the conduction band of $\mathrm{Ag}$ nanoparticles decreased nonlinearly with decreasing particle size known as the quantum confinement effect of electrons. This is because, for larger particle sizes, the number of atoms comprising the nanoparticles is enormous and its conduction band electrons are more attracted to the crystal backbone of Ag nanoparticles when compared to smaller particle sizes, thus reducing the conduction band energy with increasing particle size.

Figure 9. Conduction band of Ag nanoparticles decreased nonlinearly with increasing particle size, exclusively known as the quantum confinement effect, owing to the conduction band electrons that are more attracted to the crystal backbone of larger particle sizes.

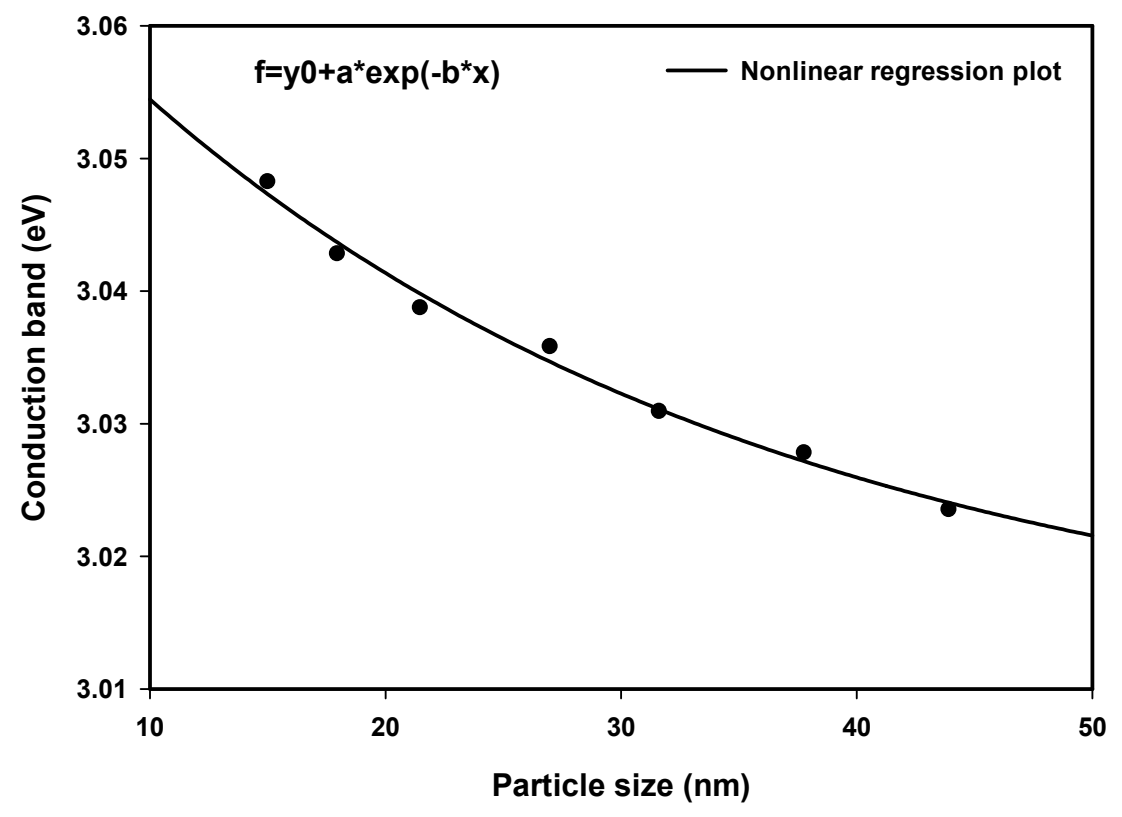

\section{Experimental Section}

Silver nitrate, $\mathrm{AgNO}_{3}$ (99\%), was used as a precursor, polyvinyl alcohol (PVA) (MW 10,000) as a capping agent, isopropanol as a radical scavenger of hydroxyl radicals, and deionized water as a solvent. All the chemical reagents were of research grade supplied by Sigma-Aldrich, St. Louis, Missouri, USA and used without further purification. The 5-wt\% PVA stock solution was made by dissolving $14.0 \mathrm{~g}$ PVA powder in $280 \mathrm{~mL}$ deionized water at $90{ }^{\circ} \mathrm{C}$. The solution was magnetically stirred for $3 \mathrm{~h}$ and was bubbled with nitrogen gas (99.5\%) in order to remove oxygen. The Ag ions concentration of $4.2 \times 10^{-4} \mathrm{M}$ together with $1 \mathrm{~mL}$ isopropanol was added into PVA solution and stirred for $2 \mathrm{~h}$. The sample was prepared and stored in a dark room to avoid reduction by light. The bulk sample was divided into seven parts and each irradiated at different doses of 10 to $70 \mathrm{kGy}$ using $1.25-\mathrm{MeV}^{60} \mathrm{Co} \gamma$-rays source. The radiation-induced synthesis offers additional benefits over the other conventional methods because it produces fully reduced and highly pure Ag nanoparticles free of byproducts or reducing agents.

Nanophox based on PCCS (Sympatec GmbH, D-38678 Clausthal-Zellerfeld, Germany) was used throughout this work to determine the particle size of prepared colloid Ag nanoparticles. The particle size and size distribution of some samples were also determined from TEM micrograph (Hitachi, H-7500, Tokyo, Japan). The TEM characterization was carried out at $100 \mathrm{kV}$. The optical properties of 
silver nanoparticles were characterized using UV-visible absorption spectroscopy (UV-1650PC Shimadzu, Kyoto, Japan). In the actual UV-visible absorption measurements, the monochromatic beam was split into two equal intensity beams. One beam, the sample, passed through a transparent cuvette containing the irradiated sample $\left(\mathrm{AgNO}_{3}, \mathrm{PVA}\right.$, IPA, and water); the other beam, the reference, passed through an identical cuvette containing irradiated reference sample (PVA, IPA, and water). All samples were diluted at the same dilution for the purpose of UV-visible absorption measurements. Prior to actual sample measurement, both cuvettes were filled with the irradiated sample and the irradiated reference sample to define the base line of the spectrophotometer system. This procedure is a necessity to ensure any absorption peaks produced from other radiochemical reactions would be discounted by the spectrophotometer system.

\section{Conclusions}

Silver nanoparticles stabilized in aqueous PVA solution were synthesized from a reduction of silver nitrate by the gamma radiolytic method. The average particle size decreased exponentially with an increasing dose and fitted to an empirical relation with the dose sensitivity parameters of $74.59 \mathrm{kGy}$. The absorption maximum at 410-nm absorption spectrum shifted to lower wavelength with increasing dose owing to a decrease in particle size at higher doses. Consequently, the conduction band energy derived from the absorption minimum increased with increasing particle size owing to the quantum confinement effect. The absorption spectrum of Ag nanoparticles was calculated using a spherical shell model and quantum physics principles and found that the calculated absorption maximum values are consistent with the measured results for the synthesized Ag nanoparticles. The results validate that the absorption peak of Ag nanoparticles is most probably derived from the intra-band excitations of conduction electrons from the lowest energy state of quantum numbers $(n=5, l=0)$ to higher energy states of quantum numbers $(n \geq 6 ; \Delta l=0, \pm 1 ; \Delta s=0, \pm 1)$ allowed by the quantum numbers principle. This demonstrates that the absorption spectrum of metal nanoparticles based on quantum physics can be exploited further, which can be added to the fundamental understanding of metal nanoparticles and the related fields of nanoscience and nanotechnology.

\section{Acknowledgments}

This study was supported by the Ministry of Higher Education of Malaysia under the FRGS and RUG grants. The authors would also like to thank the staff of the Faculty of Science, Universiti Putra Malaysia and the Nuclear Agency of Malaysia for contributing to this study.

\section{References}

1. Hornyak, G.L.; Tibbals, H.F.; Dutta, J.; Moor, J.J. Introduction to Nanoscience and Nanotechnology; CRC Press: New York, NY, USA, 2009.

2. Belloni, J.; Mostafavi, M. Radiation Induced Metal Clusters. Nucleation Mechanisms and Chemistry. In Metal Clusters in Chemistry; Braunstein, P., Oro, L.A., Raithby, P.R., Eds.; Wiley: New York, NY, USA, 1999; pp. 1213-1247. 
3. Zhong, C.J.; Luo, J.; Fang, B.; Wanjala, B.N.; Njoki, P.N. Nanostructured catalysts in fuel cells. Nanotechnology 2010, 21, 1-20.

4. Dadosh, T. Synthesis of uniform silver nanoparticles with a controllable size. Mater. Lett. 2009, 63, 2236-2238.

5. Chaney, S.B.; Shanmukh, S.; Dluhy, R.A.; Zhao, Y.P. Aligned Silver Nanorod Arrays Produce High Sensitivity Surface-Enhanced Raman Spectroscopy Substrates. Appl. Phys. Lett. 2005, 87, 031908.

6. Riveros, G.; Green, S.; Cortes, A.; Gomez, H.; Marotti, R.E.; Dalchiele, E.A. Silver nanowire arrays electrochemically grown into nanoporous anodic alumina templates. Nanotechnology 2006, 17, 561-570.

7. Astruc, D.; Lu, F.; Aranzaes, J.R. Nanoparticles as recyclable catalysts: The frontier between homogeneous and heterogeneous catalysis. Angew. J. Chem. Int. Ed. 2005, 44, 7852-7872.

8. Paulraj, P.; Janaki, N.; Sandhya, S.; Pandian, K. Single pot synthesis of polyaniline protected silver nanoparticles by interfacial polymerization and study its application on electrochemical oxidation of hydrazine. Colloids Surf. A Physicochem. Eng. Aspects. 2011, 377, 28-34.

9. Korotcenkov, G.; Han, S.D.; Stetter, J.R. Review of Electrochemical Hydrogen Sensors. Chem. Rev. 2009, 109, 1402-1433.

10. Shahverdi, A.R.; Fakhimi, A.; Shahverdi, H.R.; Minaian, M.S. Synthesis and effect of silver nanoparticles on the antibacterial activity of different Antibiotics against Staphylococcus aureus and Escherichia coli. Nanomed. Nanotechnol. Biol. Med. 2007, 3, 168-171.

11. Pal, S.; Kyung, Y.; Song, J.M. Does the antibacterial activity of silver nanoparticles depend on the shape of the nanoparticle? A study of the gram-negative bacterium Escherichia coli. Appl. Environ. Microbiol. 2007, 73, 1712-1720.

12. Rastogi, P.K.; Ganesan, V.; Krishnamoorthi, S. Microwave assisted polymer stabilized synthesis of silver nanoparticles and its application in the degradation of environmental pollutants. Mat. Sci. Eng. B. 2012, 177, 456- 461.

13. Schrand, A.M.; Braydich-Stolle, L.K.; Schlager, J.J.; Dai, L.; Hussain, S.M. Can silver nanoparticles be useful as potential biological labels? Nanotechnology 2008, 19, 235104-235116.

14. Dhar, S.; Reddy, E.M.; Shiras, A.; Pokharkar, V.; Prasad, B.L.V. Natural gum reduced/stabilized gold nanoparticles for drug delivery formulations. Chem. Eur. J. 2008, 14, 10244-10250.

15. Moskovits, M. Surface-enhanced spectroscopy. Rev. Mod. Phys. 1985, 57, 783-826.

16. Sanpui, P.; Chattopadhyay, A.; Ghosh, S.S. Induction of apoptosis in cancer cells at low silver nanoparticle concentrations using chitosan nanocarrier. ACS Appl. Mater. Interfaces 2011, 3, 218-228.

17. Jensen, T.R.; Malinsky, M.D.; Haynes, C.L.; van Duyne, R.P. Nanosphere lithography: Tunable localized surface Plasmon resonance spectra of silver nanoparticles. J. Phys. Chem. B. 2000, 104, 10549-10556.

18. Cuenya, B.R. Synthesis and catalytic properties of metal nanoparticles: Size, shape, support, composition, and oxidation state effects. Thin Solid Films 2010, 518, 3127-3150.

19. Sau, T.K.; Rogach, A.L. Nonspherical noble metal nanoparticles: Colloid-chemical synthesis and morphology control. Adv. Mater. 2010, 22, 1781-1804. 
20. Zhang, Q.; Xie, J.; Yu. Y.; Lee, J.Y. Monodispersity control in the synthesis of monometallic and bimetallic quasi-spherical gold and silver nanoparticles. Nanoscale 2010, 2, 1962-1975.

21. Kumar, A.; Joshi, H.; Pasricha, R.; Mandale, A.B.; Sastry, M. Phase transfer of silver nanoparticles from aqueous to organic solutions using fatty amine molecules. J. Colloid Interface Sci. 2003, 264, 396-401.

22. Pal, A.; Shah, S.; Devi, S. Synthesis of $\mathrm{Au}, \mathrm{Ag}$ and $\mathrm{Au}-\mathrm{Ag}$ alloy nanoparticles in aqueous polymer solution. Colloids Surf. A 2007, 302, 51-57.

23. Rodríguez-Sánchez, L.; Blanco, M.C.; López-Quintela, M.A. Electrochemical Synthesis of Silver Nanoparticles. J. Phys. Chem. B 2000, 104, 9683-9688.

24. Rivas, L.; Sanchez-cartos, S.; Garcia-Ramos, J.V.; Marcillo, G. Growth of silver colloidal particles obtained by citrate reduction to increase the Raman enhancement factor. Langmuir 2001, 17, 574-577.

25. Pastoriza-Santos, I.; Liz-Marzan, L.M. Reduction of silver nanoparticles in DMF. Formation of monolayers and stable colloids. Pure Appl. Chem. 2000, 72, 83-90.

26. Chaudhari, V.R.; Haram, S.K.; Kulshreshtha, S.K. Micelle assisted morphological evolution of nanoparticles. Colloids Surf. A 2007, 301, 475-480.

27. Hirai, H.; Nakao, Y.; Toshima, N. Preparation of Colloidal Transition Metals in Polymers by Reduction with Alcohols or Ethers. J. Macromol. Sci. Chem. 1979, 13, 633-649.

28. Silvert, P.Y.; Vijaykrishnan, V.; Vibert, P.; Herrera-Urbina, R.; Elhsissen, K.T. Synthesis and characterization of nanoscale Ag-Pd alloy particles. Nanostruct. Mater. 1996, 7, 611-618.

29. Zhou, Y.; Yu, S.H.; Wang, C.Y.; Li, X.G.; Zhu, Y.R.; Dendrites, Z.Y. A novel ultraviolet irradiation photoreduction technique for the preparation of single-crystal $\mathrm{Ag}$ nanorods and $\mathrm{Ag}$ dendrites. Adv. Mater. 1999, 11, 850-852.

30. Sudeep, P.K.; Kamat, P.V. Photosensitized Growth of Silver Nanoparticles under Visible Light Irradiation: A Mechanistic Investigation. Chem. Mater. 2005, 17, 5404-5410.

31. Tu, W.; Liu, H. Continuous synthesis of colloidal metal nanoclusters by microwave irradiation. J. Chem. Mater. 2000, 12, 564-567.

32. Vasileva, P.; Donkova, B.; Karadjova, I.; Dushkin, C. Synthesis of starch-stabilized silver nanoparticles and their application as a surface plasmon resonance-based sensor of hydrogen peroxide. Colloids Surf. A Physicochem. Eng. Aspects. 2011, 382, 203-210.

33. Mafune, F.; Kohno, J.Y.; Takeda, Y.; Kondow, T. Structure and stability of silver nanoparticles in aqueous solution produced by laser ablation. J. Phys. Chem. B 2000, 104, 8333-8337.

34. Rao, Y.N.; Banerjee, D.; Datta, A. Gamma irradiation route to synthesis of highly re-dispersible natural polymer capped silver nanoparticles. Radiat. Phys. Chem. 2010, 79, 1240-1246.

35. Temgire, M.K.; Joshi, S.S. Optical and structural studies of silver nanoparticles. Radiat. Phys. Chem. 2004, 71, 1039-1044.

36. Wu, W.T.; Wang, Y.; Shi, L.; Zhu, Q.; Pang, W.; Xu, G.; Lu, F. Fabrication of silver/cross-linked poly(vinyl alcohol) cable-like nanostructures under $\gamma$-ray irradiation. Nanotechnology 2005, 16, 3017-3022.

37. Kumara, M.; Varshney, L.; Francis, S. Radiolytic formation of Ag clusters in aqueous polyvinyl alcohol solution and hydrogel matrix. Radiat. Phys. Chem. 2005, 73, 21-27. 
38. Ramnani, S.P.; Biswal, J.; Sabharwal, S. Synthesis of silver nanoparticles supported on silica aerogel using gamma radiolysis. Radiat. Phys. Chem. 2007, 76, 1290-1294.

39. Naghavi, K.; Saion, E.; Rezaee, K.; Yunus, W.M. Influence of dose on particle size of colloidal silver nanoparticles synthesized by gamma radiation. Radiat. Phys. Chem. 2010, 79, 1203-1208.

40. Jovanovi, Z.; Krkljes, A.; Stojkovska, J.; Tomic, S.; Obradovic, B.; Miskovic-Stankovic, V.; Kacarevic-Popovic, Z. Synthesis and characterization of silver/poly(n-vinyl-2-pyrrolidone) hydrogel nanocomposite obtained by in situ radiolytic method. Radiat. Phys. Chem. 2011, 80, 1208-1215.

41. Biswal, J.; Misra, M.; Borde, L.C.; Sabharwal, S. Synthesis of silver nanoparticles in methacrylic acid solution by gamma radiolysis and their application for estimation of dopamine at low concentration. Radiat. Phys. Chem. 2012, doi:10.1016/j.radphyschem.2012.10.003.

42. Juby, K.A.; Dwivedi, C.; Kumar, M.; Kota, S.; Misra, H.S.; Bajaj, P.N. Silver nanoparticle-load PVA/gum acacia hydrogel: Synthesis, characterization and antibacterial study. Carbohydr. Polym. 2012, 89, 906-913.

43. Lai, T.; Park, H.G.; Choi, S.H. $\gamma$-Irradiation-induced preparation of Ag and Au nanoparticles and their characterizations. Mater. Chem. Phys. 2007, 105, 325-330.

44. Belloni, J. Nucleation, growth and properties of nanoclusters studied by radiation chemistry application to catalysis. Catal. Today 2006, 113, 141-156.

45. Mallicka, K.; Witcombb, M.J.; Scurrella, M.S. Self-assembly of silver nanoparticles in a polymer solvent: Formation of a nanochain through nanoscale soldering. Mater. Chem. Phys. 2005, 90, 221-224.

46. Zhiqiang, L.; Xiaobin, L.; Zhihong, P. The mechanism of agglomeration and control in the process of ultrafine powder prepared by wet chemical method. Chemistry 1999, 7, 54-57.

47. Ulanski, P.; Bothe, E.; Rosiak, J.M.; von Sonntag, C. OH radical induced crosslinking and strand breakage of poly(vinyl alcohol) in aqueous solution in the absence and presence of oxygen. A pulse radiolysis and product study. Macromol. Chem. Phys. 1994, 195, 1443-1461.

48. Abargues, R.; Gradess, R.; Canet-Ferrer, J.; Abderrafi, K.; Valdes, J.L.; Martinez-Pastor, J. Scalable heterogeneous synthesis of metallic nanoparticles and aggregate with polyvinyl alcohol. New J. Chem. 2009, 33, 913-917.

49. Zhou, F.; Zhou, R.; Hao, X.; Wu, X.; Rao, W.; Chen, Y.; Gao, D. Influences of surfactant (PVA) concentration and $\mathrm{pH}$ on the preparation of copper nano-particles by electron beam irradiation. Radiat. Phys. Chem. 2008, 77, 169-173.

50. Geers, H.; Witt, W. Direct Calculation of the Volume Based Particle Size Distribution from PCS or PCCS Measurements. In Proceedings of Particulate Systems Analysis 2008, Stratford-upon-Avon, UK, 21-23 September 2008.

51. Abedini, A.; Larki, F.; Saion, E.; Zakaria, A.; Hussein, M.Z. Influence of dose and ion concentration on formation of binary Al-Ni alloy nanoclusters. Radiat. Phys. Chem. 2012, 81, 1653-1658.

52. Mie, G. Contributions to the optics of turbid media, particularly of colloidal metal solutions. J. Ann. Phys. 1908, 25, 377-445. 
53. Noguez, C. Optical properties of isolated and supported metal nanoparticles. Opt. Mater. 2005, 27, 1204-1211.

54. Okamoto, T.; Yamaguchi, I. Optical absorption study of the surface Plasmon resonance in gold by self-assembly technique. J. Phys. Chem. B 2003, 38, 10321-10324.

55. Renteria, V.M.; Garcia-Macedo, J. Modeling of optical absorption of silver prolate nanoparticles stabilized by Gemini surfactant. Colloids Surf. A 2006, 273, 1-3.

56. Gharibshahi, E.; Saion, E. Quantum mechanical calculation of optical absorption of silver and gold nanoparticles by density functional theory. Phys. Int. 2010, 1, 57-64.

57. Saion, E.; Gharibshahi, E. On the theory of metal nanoparticles based on quantum mechanical calculation. J. Fund. Sci. 2011, 7, 6-11.

58. Gharibshahi, E.; Saion, E. Influence of dose on particle size and optical properties of colloidal platinum nanoparticles. Int. J. Mol. Sci. 2012, 13, 14723-14741.

59. Thomas, L.H. The calculation of atomic fields. Math. Proc. Camb. Philos. Soc. 1927, 23, 542-548.

60. Fermi, E. Un metodo statistico per la determinazione di alcune priorieta dell'atome. Rend. Accad. Naz. Lincei 1927, 6, 602-607.

61. Hohenberg, P.; Kohn, W. Inhomogeneous electron gas. Phys. Rev. 1964, 136, 864-871.

62. Kohn, W.; Sham, L.J. Self-consistent equations including exchange and correlation effects. Phys. Rev. 1965, 140, 1133-1138.

63. Dirac, P.A.M. Note on exchange phenomena in the Thomas atom. Math. Proc. Camb. Philos. Soc. 1930, 26, 376-385.

64. Von Weizsacker, C.F. Zur Theorie der Kernmassen. Zeitschrift fur Phys. 1935, 96, 431-458.

(C) 2013 by the authors; licensee MDPI, Basel, Switzerland. This article is an open access article distributed under the terms and conditions of the Creative Commons Attribution license (http://creativecommons.org/licenses/by/3.0/). 